\title{
A Guardian Angel Phosphatase for Mainline Carbon Metabolism
}

\author{
Guillaume A. Beaudoin ${ }^{1}$ and Andrew D. Hanson ${ }^{1, *, @ ~}$ \\ ${ }^{1}$ Horticultural Sciences Department, University of Florida, Gainesville, FL 32611, USA \\ ${ }^{*}$ Correspondence: adha@ufl.edu (A.D. Hanson) \\ @Twitter: @ADHansonLab
}

Keywords: Metabolite damage, metabolite repair, side-reaction, phosphatase, glycolysis

\begin{abstract}
It is increasingly clear that many metabolic enzymes mistakenly form minor but toxic sideproducts that must be eliminated to maintain normal fluxes. Collard et al. show that this is true of two iconic glycolytic enzymes, and that a hitherto somewhat mysterious phosphatase rescues central carbon metabolism from their mistakes.

Metabolic pathways do not come any older, more mainline, or better studied than glycolysis [1]. So glycolysis is often thought to be fully understood. However, there is much more to it than the textbooks would suggest.
\end{abstract}

A recent landmark paper by Collard et al. [2] illustrates just how much more there is to glycolysis. Using a powerful blend of mammalian and yeast biochemistry, genetics, and metabolomics, Collard et al. elegantly (i) uncover in-vivo side-reactions of two glycolytic enzymes that produce toxic phosphoesters and (ii) show that a previously rather enigmatic enzyme - phosphoglycolate phosphatase (PGP) [3] - is responsible for hydrolyzing and hence detoxifying these side-products.

The enzymatic side-reactions demonstrated by Collard et al. are the production of 4-phosphoerythronate by glyceraldehyde 3-phosphate dehydrogenase and the production of 2-phospho-L-lactate by pyruvate kinase (Figure 1A). 4-Phosphoerythronate inhibits the pentose phosphate pathway enzyme 6-phosphogluconate dehydrogenase, and 2-phospho-L-lactate inhibits phosphofructokinase-2, the enzyme that makes the glycolytic activator fructose-2,6-bisphosphate (Figure 1A). When PGP was knocked out, mammalian cells accumulated 4-phosphoerythonate, whose buildup provoked massive accumulation of 6-phosphogluconate. The knockout cells also accumulated 2-phospho-L-lactate and consequently became depleted in fructose-2,6-bisphosphate. Together, these changes led to severe knock-on effects on flux through the pentose phosphate and glycolysis pathways. Analogous results were obtained in yeast when its PGP homolog (Pho13) was deleted. Thus, without their guardian angel phosphatase, two mainline carbon pathways go seriously off-track in mammals and yeast. 
The discoveries of these glycolytic side-reactions and the corresponding enzymatic 'fix' fit into a wider conceptual framework that is gaining increasing recognition [4,5]. According to this framework, metabolites are subject to enzymatic mistakes and to spontaneous chemical reactions that lead to side-products that are always wasteful and sometimes toxic. These unwanted metabolic side-events are termed 'metabolite damage'. Cells deal with this damage using two types of damage-control system: 'damage repair' enzymes such as PGP correct damage after it happens, and 'damage preemption' enzymes reduce the chance of it happening in the first place, for example, by reducing the pool size of damage-prone compounds [4]. The general concepts of metabolite damage and metabolite damage-repair are illustrated in Figure 1B. Note that these concepts parallel the far better established concepts of DNA and protein damage and damage-repair [4].

PGP and Pho13 are from mammals and yeast but there are analogous examples, from other kingdoms of life, of phosphatases that repair damage from reactions involving intermediates of central carbon metabolism. These repair phosphatases include CbbY [6] (from plants and bacteria) and CA1Pase [7] (from plants), which hydrolyze different bisphosphate side-products ('misfire products') of the $\mathrm{CO}_{2}$-fixing enzyme Rubisco; the side-products are potent Rubisco inhibitors. Other examples are DUF89 family enzymes (from plants and yeast) that hydrolyze aberrant sugar phosphates [8].

The demonstration by Collard et al. that mammalian and fungal glyceraldehyde 3-phosphate dehydrogenase and pyruvate kinase mediate problematic side-reactions in vivo raises two questions: Do the cognate plant and prokaryote enzymes mediate the same side-reactions? And if so, do these organisms have functional equivalents of PGP and Pho13? In this connection, it is worth noting that the model plant Arabidopsis thaliana has two homologs of PGP and Pho13: AtPGLP1, which is the photorespiratory phosphoglycolate phosphatase, and AtPGLP2, whose function is unknown [9]. Likewise, Escherichia coli has a phosphoglycolate phosphatase (Gph) whose physiological function is far from clear [10]. Could these plant and bacterial enzymes be PGP/Pho13 equivalents?

A final - and crucial - point underscored by the Collard et al. study is that the side reactions of enzymes do not have to be at all prominent in order to be physiologically devastating when not kept in check. Thus, the catalytic efficiencies of the side-reactions of glyceraldehyde 3-phosphate dehydrogenase and pyruvate kinase are thousands of times lower than that of their respective canonical reactions and in vivo fluxes through the side reactions are tiny compared to the canonical reaction fluxes. These in vivo flux trickles nevertheless generate seriously inhibitory accumulations of damage products in the absence of PGP or Pho13. A historical consequence of this mismatch between enzymological and physiological perspectives is that side-reactions of enzymes that seem negligible in the test tube, or are not readily detected in standard assay conditions, were in the past easily but 
erroneously dismissed as insignificant and uninteresting. May the paper by Collard et al. warn metabolic biochemists against perpetuating this unfortunate error.

\section{Acknowledgments}

This work was supported by U.S. National Science Foundation award MCB 1611711 to A.D.H.

\section{References}

1. Bar-Even, A. et al. (2012) Rethinking glycolysis: on the biochemical logic of metabolic pathways. Nat. Chem. Biol. 8, 509-517

2. Collard, F. et al. (2016) A conserved phosphatase destroys toxic glycolytic side products in mammals and yeast. Nat. Chem. Biol. 12, 601-607

3. Badwey, J.A. (1977) Phosphoglycolate phosphatase in human erythrocytes. J. Biol. Chem. 252, 2441-2443

4. Linster, C.L. et al. (2013) Metabolite damage and its repair or pre-emption. Nat. Chem. Biol. 9, $72-80$

5. Hanson, A.D. et al. (2016) Metabolite damage and metabolite damage control in plants. Annu. Rev. Plant Biol. 67, $131-152$

6. Bracher, A. et al. (2015) Degradation of potent Rubisco inhibitor by selective sugar phosphatase. Nat. Plants 1, 14002

7. Andralojc, P.J. et al. (2012) 2-Carboxy-d-arabinitol 1-phosphate (CA1P) phosphatase: evidence for a wider role in plant Rubisco regulation. Biochem. J. 442, 733-742

8. Huang, L. et al. (2016) A family of metal-dependent phosphatases implicated in metabolite damage-control. Nat. Chem. Biol. 12, 621-627

9. Schwarte, S. and Bauwe, H. (2007) Identification of the photorespiratory 2-phosphoglycolate phosphatase, PGLP1, in Arabidopsis. Plant Physiol. 144: 1580-1586

10. Pellicer, M.T. et al. (2003) Role of 2-phosphoglycolate phosphatase of Escherichia coli in metabolism of the 2-phosphoglycolate formed in DNA repair. J. Bacteriol. 185, 5815-5821

\section{Figure legend}

Figure 1. Metabolite Damage and Repair. (A) The mistakes made by the promiscuous enzymes glyceraldehyde 3-phosphate dehydrogenase and pyruvate kinase (highlighted in rose) give rise to damaged metabolites (boxed in red) that are inhibitory. The repair enzyme PGP (highlighted in blue) dephosphorylates the damaged metabolites, rendering them harmless. (B) The general principles of metabolite damage and repair. Damage can be caused by a promiscuous (sloppy) enzyme acting on non-canonical substrate $X$ instead of its canonical substrate $A$, giving rise to damage product $Y$. A repair enzyme removes $Y$, converting it to innocuous product $Z$. 
Figure 1

(A)

\section{Glucose}

6-P-Gluconolactone

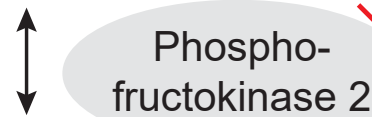

6-P-Gluconate

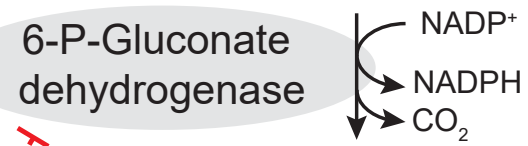

Ribulose-5-P

Fructose-6-P $\longrightarrow$ Fructose-2,6-BP

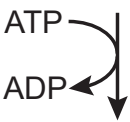
ATP ADP

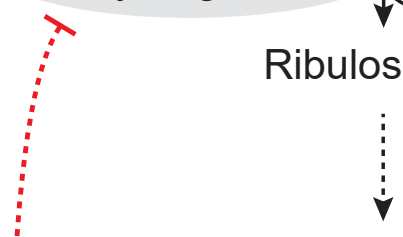

Erythrose-4-P

Fructose-1,6-BP

Glyceraldehyde-3-P

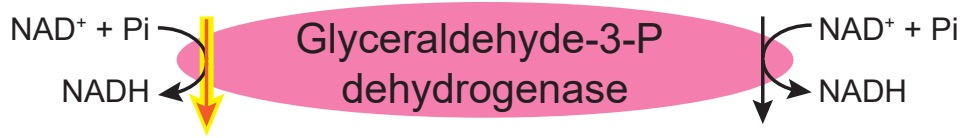

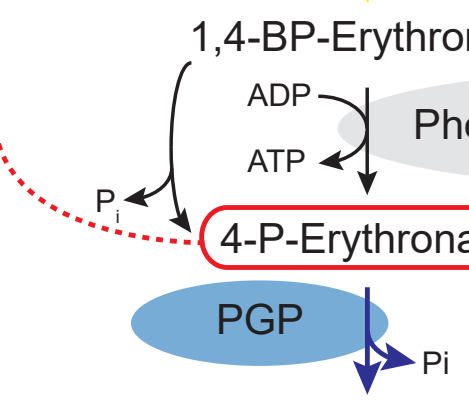

Erythronate

1,3-BP-Glycerate

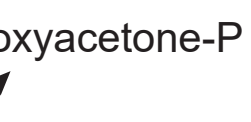

$$
\text { (i) }
$$

